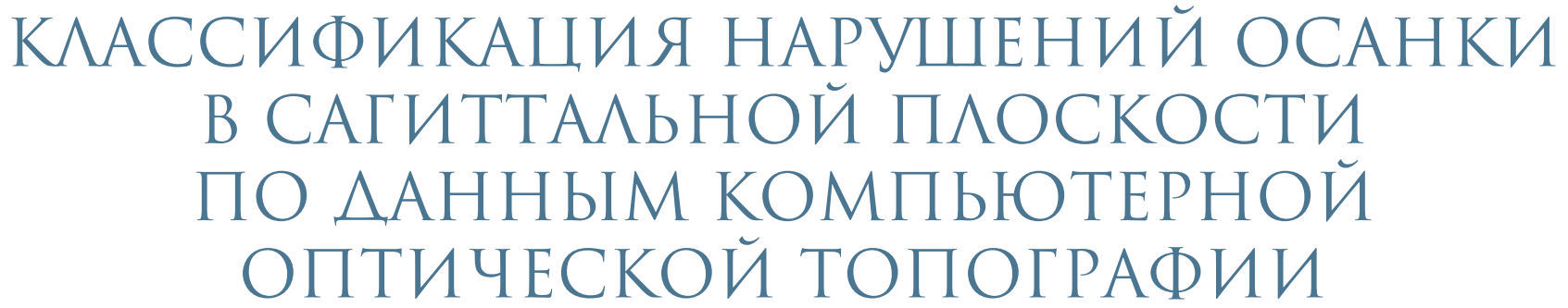

В.Н. Сарнадскии́

ООО «Медииинские топографические системы «МЕТОС», Новосибирск

\begin{abstract}
Цель исследования. Изучение по данным компьютерной оптической топографии (КОМОТ) возможных сочетаний выраженности физиологических изгибов позвоночника (поясничного лордоза и грудного кифоза) и создание уточненной классификации нарушений осанки в сагиттальной плоскости, основанной на статистически обоснованных топографических нормах и критериях.

Материал и методы. По результатам скринига детей и подростков методом КОМОТ в шести городах собрана клиническая база, включающая более 33000 пациентов с приблизительно равномерным распределением по возрасту (от 4,5 до 17,5 лет).

Результаты. Разработана и введена в программное обеспечение системы ТОДП (WTOPO версия 3.8-11) новая (уточненная) классификация нарушений осанки в сагиттальной плоскости по данным метода КОМОТ, которая дифференцирует нарушения осанки по тяжести на слабовыраженные, умеренно выраженные и выраженные отклонения, учитывает все возможные варианты соотношения по выраженности физиологических дуг. Классификация содержит 21 тип осанки: гармоничную осанку, 8 типов для слабовыраженных нарушений, 7 - для умеренных, 5 - для выраженных, что позволит по результатам топографических обследований более точно дифференцировать состояние осанки в сагиттальной плоскости.

Заключение. Предложенная классификация нарушений осанки в сагиттальной плоскости важна как для клинической практики, так и для научных исследований.

Ключевые слова: компьютерная оптическая топография, классификация нарушений осанки в сагиттальной плоскости.
\end{abstract}

\section{CLASSIFICATION OF SAGITTAL PLANE POSTURE DISORDERS AS EVIDENCED BY COMPUTER OPTICAL TOPOGRAPHY \\ V.N. Sarnadsky}

Objective. To study possible combinations of severity of physiological spinal curvatures (lumbar lordosis and thoracic kyphosis) evidenced by computer optical topography (COMOT), and to elaborate specified classification of sagittal plane posture disorders based on statistically valid topographic norms and criteria.

Material and Methods. Results of the COMOT screening of children and adolescents in six cities formed a clinical database including more than 33,000 patients approximately evenly distributed through ages: ranged from 4.5 to 17.5 years.

Results. A new classification of sagittal plane posture disorders was elaborated based on the COMOT method findings. This classification differentiates posture disorders on manifestation of deviations into mild, moderate, and marked considering all possible relations of severity of physiological curvatures. It contains 21 posture types: a harmonic, 8 types of mild disorders, 7 types of moderate disorders, and 5 types of marked disorders.

Conclusion. In 2011, the proposed classification of sagittal plane posture disorders was introduced into the TODP software system ( WTOPO version 3.8-11), that would allow for more accurate differentiation of sagittal plane posture based on topography survey results. This is important for both clinical practice and scientific research.

Key Words: computer optical topography, classification, posture disorders, sagittal plane.

Hir. Pozvonoc. 2011; (3):46-55.
В 2004 г. в рамках реализации программы правительства Москвы «Диагностика и коррекция нарушений осанки у школьников» (2003-2005 гг.) автор совместно с московскими врачами- ортопедами, участниками программы, для интерпретации результатов скрининг-обследования на системе ТОДП [1] разработал формализованный топографический диагноз для оценки состояния осанки в трех плоскостях: фронтальной, сагиттальной и горизонтальной [3]. Указанный диагноз прошел апробацию при обследовании 23 тыс. учащихся 1-х и 6-х классов Южного 
административного округа Москвы и Зеленограда и получил одобрение со стороны организаторов и участников этой программы, в том числе главного детского ортопеда Министерства здравоохранения и социального развития проф. О.А. Малахова и ведущего специалиста Министерства здравоохранения и социального развития по спортивной медицине и реабилитации проф. Б.А. Поляева.

В основе топографического диагноза лежит понятие гармоничной осанки [3], критерии которой следующие:

- оптимальная статика туловища во фронтальной и сагиттальной плоскостях;

- симметрия туловища относительно срединной линии;

- отсутствие боковых искривлений позвоночника;

- отсутствие скручивания туловища вдоль продольной оси в горизонтальной плоскости;

- сбалансированность физиологических изгибов и их анатомически правильное положение;

- соответствие основных параметров формы дорсальной поверхности туловища среднестатистическим значениям, полученным по результатам топографического скрининга пациентов 5-17 лет.

В зависимости от выраженности нарушений осанки ее состояние разделяют на пять уровней: «здоровые - норма» (3Н) - осанка, близкая к гармоничной; «здоровые - субнорма» (3С) - имеются слабовыраженные отклонения; «нарушения осанки» (HO) - умеренно выраженные отклонения; «деформация позвоночника» (ДП) выраженные отклонения; «деформация позвоночника» (ДП) - имеются значительные отклонения. Выраженность отклонений от гармоничного состояния осанки оценивают на основе $\sigma$-нормированных топографических параметров: $\mathrm{P}_{\sigma}=\left(\mathrm{P}-\mathrm{P}_{\mathrm{H}}\right) / \sigma_{\mathrm{p}}$, где $\mathrm{P}-$ значение топографического параметра, полученное по результатам обследования пациента; $\mathrm{P}_{\mathrm{H}}-$ значение нормы для данного параметра (среднестатистическое значение параметра для детей и подростков 5-17 лет); $\sigma_{\mathrm{p}}-$ сред- неквадратическое отклонение [2]. 3начение параметра $\mathrm{P}_{\sigma}$ от $-2 / 3$ до $+2 / 3$ соответствует гармоничной осанке от $-2 / 3$ до - 1 и от $+2 / 3$ до $+1-$ субнорме; от - 1 до - 2 и от +1 до $+2-$ умеренным отклонениям; от -2 до -3 и от +2 до +3 - выраженным отклонениям; от -3 и менее и от +3 и более - значительным отклонениям. При этом отрицательные значения $\mathrm{P}_{\sigma}$ соответствуют уменьшению параметра Р по сравнению с нормой, а положительные - увеличению (например, при оценке выраженности грудного кифоза и поясничного лордоза отрицательные значения $\mathrm{P}_{\sigma}$ соответствуют уплощению, а положительные - усилению физиологических изгибов). Для облегчения интерпретации топографических данных на системе ТОДП используют раскраску параметров по принципу светофора: зеленый цвет соответствует норме и субнорме (зеленая зона - отклонение от нормы не более $\pm \sigma)$; желтый умеренным отклонениям (желтая зона - отклонение от нормы от $\pm \sigma$ до $\pm 2 \sigma)$; красный - выраженным отклонениям (красная зона - отклонение от нормы более $\pm 2 \sigma$ ).

При использовании $\sigma$-нормировки предполагалось, что топографические параметры, подобно антропометрическим, имеют нормальный закон распределения, при котором значению параметра в пределах $\pm 2 / 3 \sigma$ соответствует $50,00 \%$ случаев; $\pm \sigma-68,26 ; \pm 2 \sigma$ - 95,44; $\pm 3 \sigma-99,72$. Следовательно, в пределы нормы по параметру должно попадать 50,00\% случаев, субнормы - 18,26, умеренных отклонений - 27,18, выраженных отклонений 4,28, значительных отклонений $-0,28$. Наши дальнейшие исследования действительно показали, что большинство топографических параметров имеет распределение, близкое к нормальному закону, как это видно из рис. 1, на котором приведены гистограммы двух топографических параметров HIL и НIK, описывающих соответственно выраженность по глубине поясничного лордоза и грудного кифоза (в дальнейшем лордоз и кифоз). Однако установлено, что распределения большинства топографических параметров имеют асимметрию (третий момент распределения не равен 0). Так, для параметра HIL коэффициент асимметрии составил $+0,36$, а для НІК $-+0,14$, поэтому $\sigma$-нормировка HIL и НІК без учета этой асимметрии их распределения приводит к уменышению в процентном выражении случаев уплощения и увеличению числа случаев с усилением физиологических изгибов.

Использованный подход к оценке выраженности нарушений осанки позволил разработать статистически обоснованные топографические критерии и нормы для оценки результатов обследований на системе ТОДП [2], которые успешно используют на практике более 15 лет. Анализ накопленных результатов массовых топографических обследований, помимо асимметрии распределений топографических параметров, выявил и другие моменты, которые требуют учета и дальнейшей доработки формализованного топографического диагноза, в том числе и для оценки состояния осанки в сагиттальной плоскости.

При разработке формализованного топографического диагноза основным требованием со стороны ортопедов была его максимальная преемственность существующим на практике подходам, поэтому для сагиттальной плоскости использовали общепринятую отечественными ортопедами классификацию нарушений осанки, основанную на клиническом осмотре пациента врачом и визуальной оценке лордоза и кифоза. Классификация учитывает соотношение выраженности лордоза и кифоза и включает пять типов нарушений: плоская спина (НО-Пл) - уплощение кифоза и лордоза; плосковогнутая спина (НО-ПВ) - уплощение кифоза и усиление лордоза; кругловогнутая спина (НО-КВ) - усиление кифоза и лордоза; круглая спина (НОКр) - усиление кифоза при нормальном лордозе; сутулая спина (НО-Су) усиление кифоза при увеличении его протяженности и уплощении лордоза (в скобках приведено краткое обозначение типа нарушений, используемое в системе ТОДП). Перечисленные типы нарушений были отнесены 


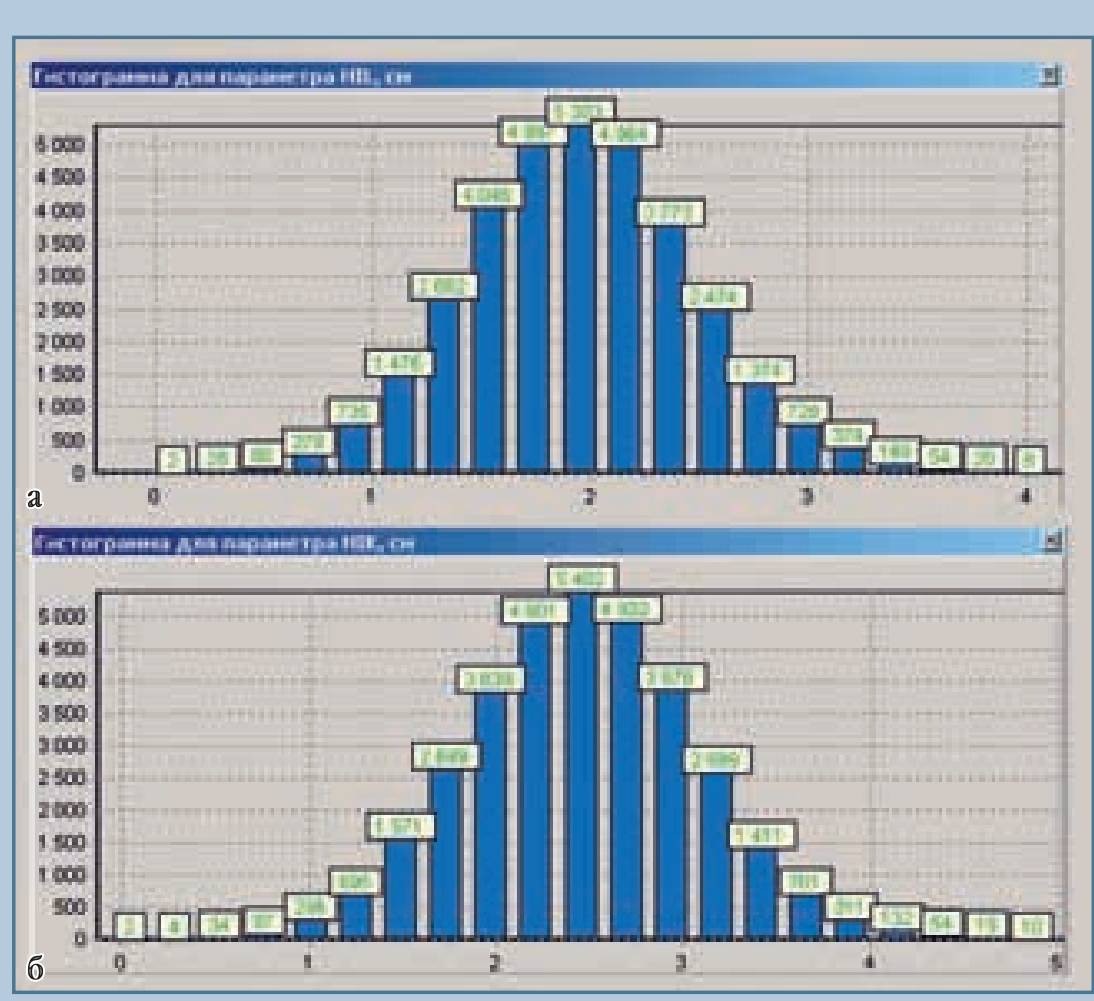

Рис. 1

Гистограммы распределения высоты дуг физиологических изгибов - поясничного лордоза (а) и грудного кифоза (б): параметры интегральной высоты лардоза (HIL) и интегральной высоты кифоза (HIK) рассчитаны по формулам (1) и (2)

к умеренным отклонениям (желтая зона). Так как в отличие от визуального осмотра топографическое обследование дает точную количественную оценку состояния осанки (в миллиметрах и градусах), то это позволяет дополнительно дифференцировать осанку пациента в зеленой зоне, а наиболее выраженные нарушения осанки обозначать как деформации позвоночника и относить их к красной зоне. Для нормы и слабовыраженных отклонений (зеленая зона) введены следующие типы нарушений: 3Н - осанка, близкая к гармоничной; 3С-Уп - субнорма с уплощением физиологических изгибов; 3С-Ус - субнорма с усилением физиологических изгибов. Нарушения при выраженных и значительных отклонениях (красная зона): ДП-Пл - плоская спина; ДП-КВ - кругловогнутая спина;
ДП-Л - гиперлордоз; ДП-К1 - кифоз І ст.; ДП-К2 - кифоз II ст. Кроме того, для умеренных отклонений в состоянии физиологических изгибов, которые нельзя отнести к определенному типу, введен дополнительный тип НО-ДН - другие нарушения.

Анализ накопленных результатов массовых топографических обследований выявил, что более 16\% пациентов относятся к типу НО-ДН. Более половины таких случаев составляют пациенты с уплощенным в желтой зоне лордозом и нормальным кифозом, около трети - пациенты, имеющие промежуточное состояние между плосковогнутой и кругловогнутой спиной (усиленный лордоз при нормальном кифозе), остальные случаи - пациенты с нормальными по величине лордозом и кифозом, имеющие нарушенный баланс туловища в сагиттальной плоскости (чрезмерный наклон кпереди или кзади) или нарушение уровня расположения по продольной оси туловища вершин кифоза и лордоза или границы между ними. Для зеленой зоны оказалось явно недостаточно наличия только двух типов нарушений (с уплощением и усилением лордоза и кифоза), так как на практике чаще встречаются несбалансированные физиологические изгибы с превалированием одного из них. Указанные обстоятельства потребовали уточнения и дальнейшей доработки классификации нарушений осанки в сагиттальной плоскости для интерпретации результатов обследований пациентов на системе ТОДП, а также уточнения используемых ранее норм и критериев.

Цель исследования - изучение по данным компьютерной оптической топографии (КОМОТ) возможных сочетаний выраженности физиологических изгибов позвоночника (поясничного лордоза и грудного кифоза) и создание уточненной классификации нарушений осанки в сагиттальной плоскости, основанной на статистически обоснованных топографических нормах и критериях.

\section{Материал и методы}

В рамках настоящего исследования использована клиническая база скрининг-обследований «МЕТОС (КБСОМ), которая специально создана автором для научных исследований. В КБСОМ вошли результаты скрининг-обследований детского населения на системах ТОДП второго поколения (с фиксированной оптической схемой) в шести городах: Абакане, Новосибирске, Омске, Перми, Тольятти и Тюмени. База включает более 33000 пациентов: около 5500 человек на каждый город, с примерно равномерным распределением по полу и возрасту (от 4,5 до 17,5 лет); общее число мальчиков - 16576 , средний возраст 11,11 \pm 3,26 года; общее число девочек - 16948, средний во3раст 11,22 \pm 3,33 года. Из исходных баз удалены все некорректные снимки пациентов, а некорректно обработанные - переобработаны. 

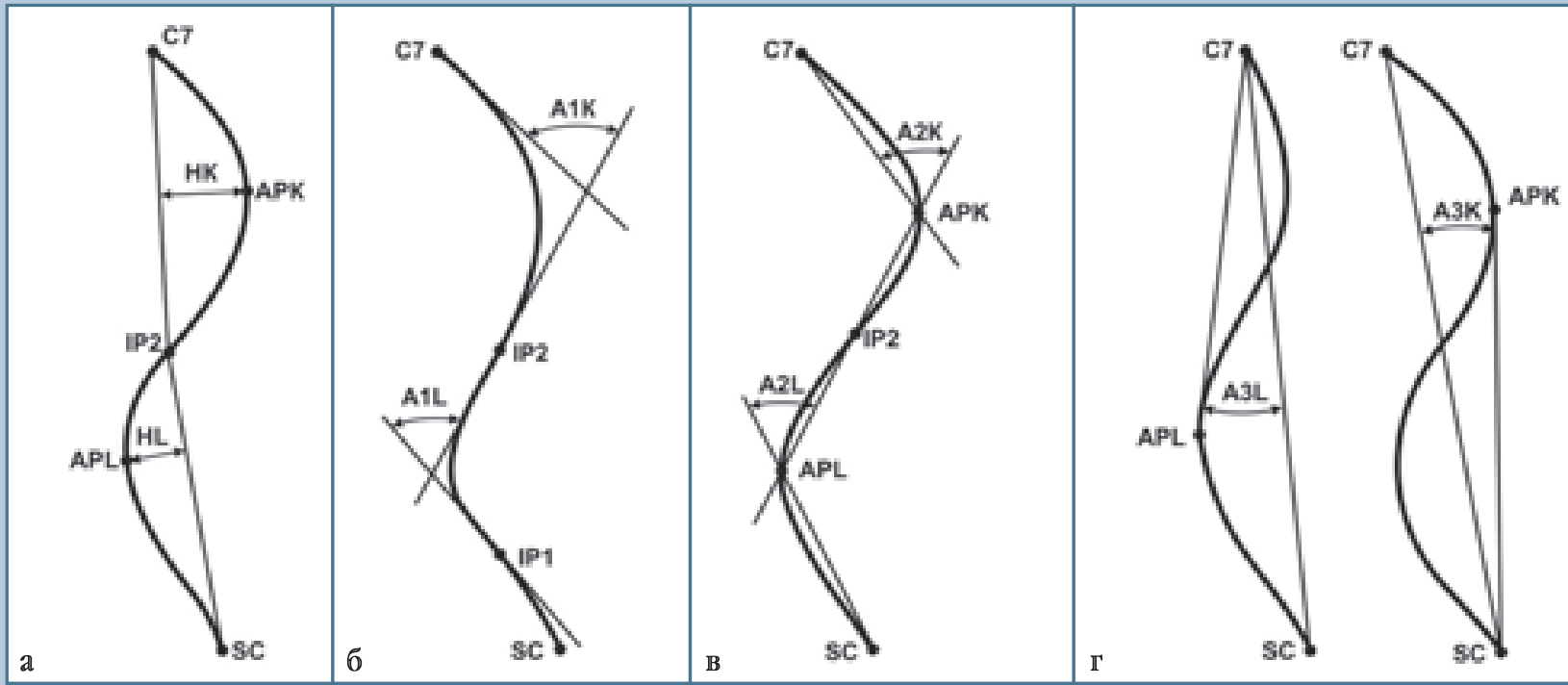

Рис. 2

Способы определения топографических параметров оценки выраженности кифоза и лордоза: а - высоты дуг; б - описанных углов раскрытия дуг; в - вписанных углов раскрытия дуг; г - проективных углов дуг; С7 - вершина остистого отростка седьмого шейного позвонка; SC - вершинная точка межъягодичной складки; APK, APL - вершины (апексы) дуг кифоза и лордоза; IP1, IP2 - точки перегиба сагиттального профиля на границе «крестец - лордоз» и «лордоз - кифоз»; HK, HL - высота (глубина) дуги кифоза и лордоза; A1K, A1L - описанные углы раскрытия кифоза и лордоза; A2K, A2L - вписанные углы раскрытия кифоза и лордоза; АЗК, АЗL - проективные углы кифоза и лордоза

Для количественной оценки выраженности кифоза и лордоза в системе ТОДП используются четыре пары первичных параметров [2]: HK, HL - высота (глубина) дуги кифоза и лордоза; A1K, A1L - описанные углы раскрытия кифоза и лордоза; A2K, A2L - вписанные углы раскрытия кифоза и лордоза; АЗК, A3L - проективные углы кифоза и лордоза. На рис. 2 показаны способы определения указанных первичных параметров.

Для повышения достоверности и устойчивости оценки выраженности дуг физиологических изгибов в системе ТОДП в старой версии классификации использовали интегральную нормированную высоту дуг кифоза и лордоза, которую рассчитывали по четырем первичным параметрам в соответствии с формулами:

$$
\begin{aligned}
\mathrm{HIL} & =\frac{1}{2}\left(\mathrm{HL}^{*}+\mathrm{HL}_{\mathrm{H}}^{*}\left(0,2 \frac{\mathrm{A} 1 \mathrm{~L}}{\mathrm{~A} 1 \mathrm{~L}_{\mathrm{H}}}+0,4 \frac{\mathrm{A} 2 \mathrm{~L}}{\mathrm{~A} 2 \mathrm{~L}_{\mathrm{H}}}+0,4 \frac{\mathrm{A} 3 \mathrm{~L}}{\mathrm{~A} 3 \mathrm{~L}_{\mathrm{H}}}\right)\right), \\
\mathrm{HIK} & =\frac{1}{2}\left(\mathrm{HK}^{*}+\mathrm{HK}_{\mathrm{H}}^{*}\left(0,2 \frac{\mathrm{A} 1 \mathrm{~K}}{\mathrm{~A} 1 \mathrm{~K}_{\mathrm{H}}}+0,4 \frac{\mathrm{A} 2 \mathrm{~K}}{\mathrm{~A} 2 \mathrm{~K}_{\mathrm{H}}}+0,4 \frac{\mathrm{A} 3 \mathrm{~K}}{\mathrm{~A} 3 \mathrm{~K}_{\mathrm{H}}}\right)\right),
\end{aligned}
$$

где HL* (HK*) - высота дуги лордоза (кифоза), нормированная на длину туловища (расстояние от SC до С7, рис. 2) в соответствии с выражением HL* = HL·Lng $/$ Lng (Lng - длина туловища у пациента; Lng - длина туловища, для которой рассчитана норма $\mathrm{HL}_{\mathrm{H}}^{*}$ и $\mathrm{HK}_{\mathrm{H}}^{*}$ ); A1L-A3L (A1K-A3K) - углы дуги лордоза (кифоза); $\mathrm{A}_{\mathrm{H}} \mathrm{L}_{\mathrm{H}} \mathrm{A}_{\mathrm{H}}\left(\mathrm{A} 1 \mathrm{~K}_{\mathrm{H}}-\mathrm{A} 3 \mathrm{~K}_{\mathrm{H}}\right)-$ значение нормы для углов дуги лордоза (кифоза). Для новой классификации с целью коррекции выявленной асимметричности распределений HIL и HIK (как это видно из рис. 1) автором были предложены новые формулы:

$$
\begin{aligned}
\mathrm{HIL} & =\frac{1}{2}\left(\mathrm{HL}^{*}+\mathrm{HL}_{\mathrm{H}}^{*}+\sigma_{\mathrm{HL}^{*}}\left(0,2 \mathrm{~A} 1 \mathrm{~L}_{\sigma_{ \pm}}+0,4 \mathrm{~A} 2 \mathrm{~L}_{\sigma \pm}+0,4 \mathrm{~A} 3 \mathrm{~L}_{\sigma \pm}\right)\right), \\
\mathrm{HIK} & =\frac{1}{2}\left(\mathrm{HK}^{*}+\mathrm{HK}_{\mathrm{H}}^{*}+\sigma_{\mathrm{HL}^{*}}\left(0,2 \mathrm{~A} 1 \mathrm{~K}_{\sigma \pm}+0,4 \mathrm{~A} 2 \mathrm{~K}_{\sigma_{ \pm}}+0,4 \mathrm{~A} 3 \mathrm{~K}_{\sigma \pm}\right)\right),
\end{aligned}
$$

где $\sigma_{\mathrm{HL}}\left(\sigma_{\mathrm{HK}^{*}}\right)$ - среднеквадратическое отклонение нормированной к длине туловища высоты лордоза (кифоза); $\mathrm{A1L} \mathrm{L}_{\sigma \pm}$, $\mathrm{A}^{2} \mathrm{~L}_{\sigma \pm}, \mathrm{A}^{2} \mathrm{~L}_{\sigma \pm}\left(\mathrm{A} 1 \mathrm{~K}_{\sigma \pm}, \mathrm{A} 3 \mathrm{~K}_{\sigma \pm}, \mathrm{A} 3 \mathrm{~K}_{\sigma \pm}\right)$ - угловые параметры дуг лордоза и кифоза после $\sigma$-нормирования с коррекцией асим- 
метрии распределений. Для топографического параметра Р такая нормировка выполняется по формуле, предложенной автором: $\mathrm{P}_{\sigma \pm}=\left(\mathrm{P}_{\mathrm{H}}\right) /\left(\sigma_{\mathrm{p}^{*}}\left(1 \pm \mathrm{KA} \mathrm{P}_{\mathrm{P}} / 2\right)\right)$, где $\mathrm{P}_{\mathrm{H}}-$ норма (среднее значение параметра); $\sigma_{\mathrm{p}}-$ среднеквадратическое значение параметра; КА - индекс асимметрии статистического распределения параметра (третий момент распределения), полученный по КБСОМ. Для отрицательных значений разности параметра Р и его нормы Р перед КА берется знак «-», а для положительных - знак «+», что позволяет для отрицательных значений растянуть, а для положительных - сжать гистограмму, делая ее более симметричной.

Как видно из рис. 3, распределения HIL и HIK, рассчитанные по новым формулам (3) и (4), стали существенно более симметричными (коэффициент асимметрии для HIL стал равным 0,12; для HIK - 0,06, то есть для лордоза он уменышился в три раза, для кифоза - в два). Для коррекции остаточной асимметрии распределения HIK и HIL при использовании в классификаторе нарушений осанки проводят дополнительно б-нормировку этих параметров с коррекцией асимметрии распределений.

Для оценки сбалансированности дуг кифоза и лордоза по выраженности в системе ТОДП используют индекс асимметрии [2]. Для параметров HIK и HIL расчет этого индекса выглядит следующим образом:

$$
\mathrm{IHI}=10 \frac{\mathrm{HIK}-\mathrm{HIL}}{|\mathrm{HIK}|+|\mathrm{HIL}|+|\mathrm{HIN}|},
$$

где 10 - нормирующий множитель; $\|$ - знак модуля (значение берется без учета знака); HIN - полусумма нормы для высоты дуги кифоза и лордоза. Достоинство данного индекса асимметрии в том, что его знак показывает, что превалирует («+» - кифоз, «» - лордоз), а значение индекса показывает степень разбалансированности высоты дуг кифоза и лордоза. Для гармоничной осанки IHІ равен 0,6 (кифоз несколько больше лордоза); для сбалансированной - от 0,2 до 1,0; для лордозированной - меньше 0,2; для кифозированной - больше 1,0.

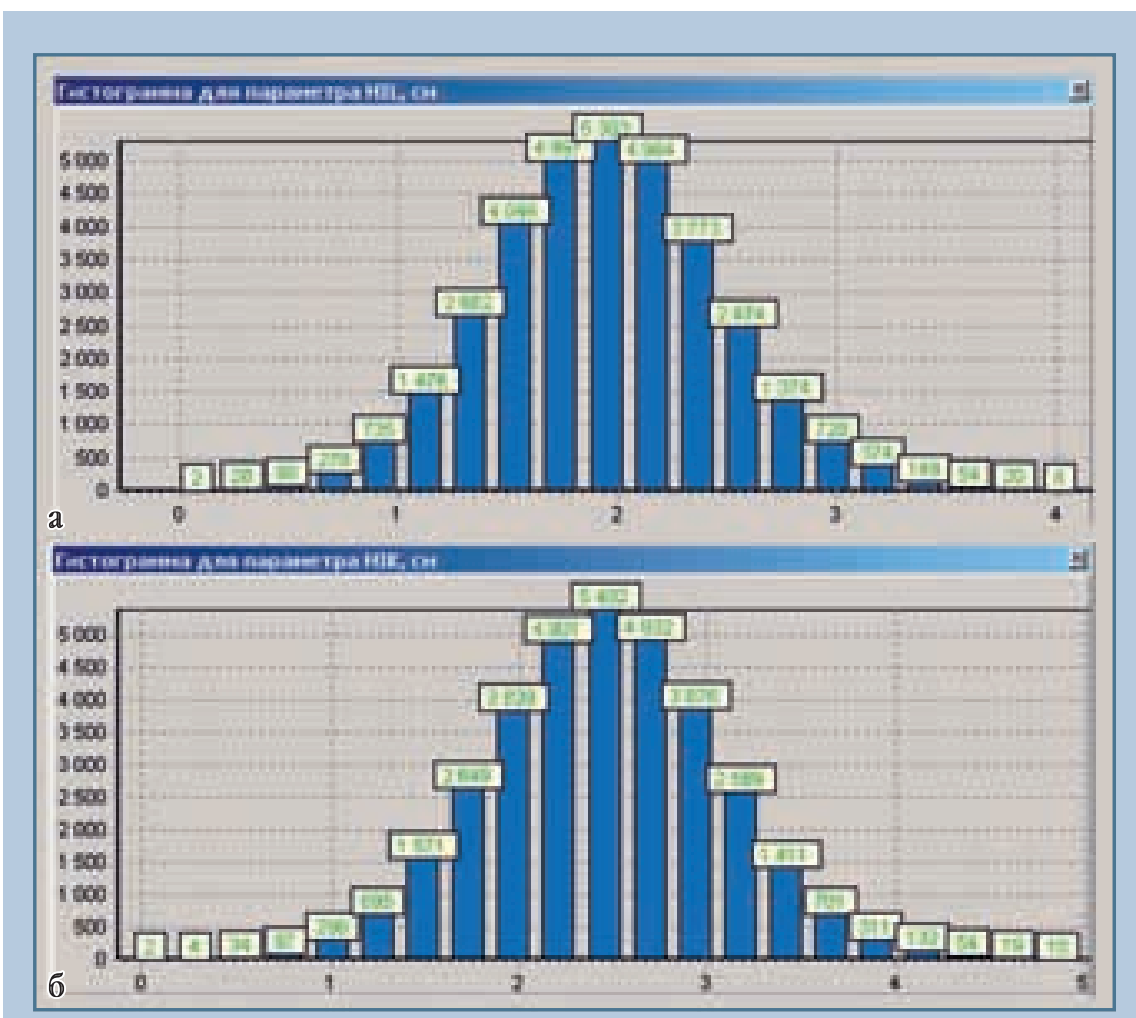

Рис. 3

Гистограммы распределения высоты дуг физиологических изгибов - поясничного лордоза (а) и грудного кифоза (б): параметры интегральной высоты лардоза (HIL) и интегральной высоты кифоза (НIK) рассчитаны по формулам (3) и (4)

\section{Результаты и их обсуждение}

Проведенные исследования позволили создать новую (уточненную) классификацию нарушений осанки в сагиттальной плоскости по данным КОМОТ. Классификация содержит гармоничную осанку и 20 типов ее нарушений: 8 типов для зеленой зоны, 7 - для желтой, 5 - для красной. Структура классификации показана на рис. 4.

По сравнению с предыдущей, в новой классификации для зеленой зоны введено 6 типов субнормы (слабовыраженных нарушений осанки): 3С-НБ - субнорма с нарушением баланса, при этом по выраженности лордоз и кифоз соответствуют гармоничному состоянию, но имеется умеренный или выраженный наклон туловища кпереди-кзади; 3С-НГ - субнорма с нарушением геометрии, при этом по выраженности лордоз и кифоз соответствуют гармоничному состоянию, но нарушено анатомически правильное положение их апексов (вершин дуг) или границы между ними; 3С-УпК - субнорма с уплощением кифоза, при этом кифоз уплощен в пределах зеленой зоны при нормальном лордозе; 3С-УсЛ - субнорма с усилением лордоза, при этом лордоз усилен в пределах зеленой зоны 


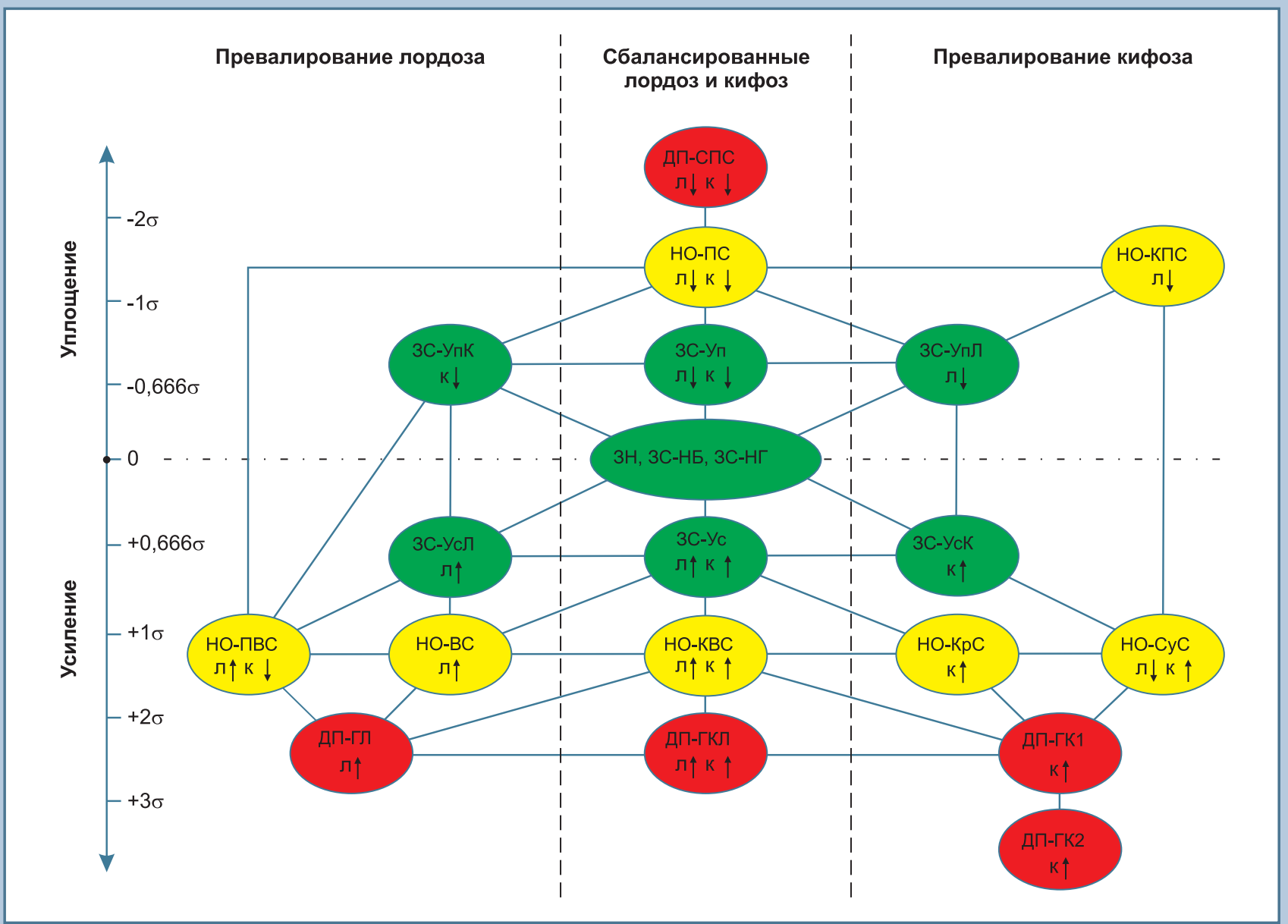

Рис. 4

Структурная схема классификации нарушений осанки в сагиттальной плоскости:

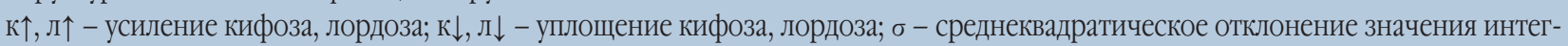
ральной нормированной высоты дуг кифоза (HIK) и лордоза (HIL); линии соединяют граничные типы нарушений осанки, то есть типы, имеющие между собой промежуточные (переходные) состояния

при нормальном кифозе; 3С-УпЛ - субнорма с уплощением лордоза, при этом лордоз уплощен в пределах зеленой зоны при нормальном кифозе; 3С-УсК - субнорма с усилением кифоза, при этом кифоз усилен в пределах зеленой зоны при нормальном лордозе.

Для желтой зоны в новой классификации дополнительно введены 2 типа: НО-КПС - круглоплоская спина, при этом имеется нормальный кифоз и уплощенный в желтой или красной зоне лордоз; НО-ВС - вогнутая спина, при этом имеется нормальный кифоз и усиленный в пределах желтой зоны лордоз, что соответствует состоянию с превалированием лордоза, занимающему промежуточное положение между плосковогнутой и кругловогнутой спиной.

Из желтой зоны упразднен тип НО-ДН, так как расширение классификации по типам позволило учесть все возможные варианты нарушений.

Для красной зоны типы нарушений осанки остались прежними, но с целью более четкого различия наименований при умеренных и выраженных нарушениях для последних введены новые обозначения и названия:
ДП-СПС - синдром прямой спины; ДП-ГКЛ - гиперкифолордоз; ДП-ГЛ - гиперлордоз; ДП-ГК1 - гиперкифоз I ст.; ДП-ГК2 - гиперкифоз II ст.

В табл. 1 приведены краткое описание каждого из 21 типа осанки в сагиттальной плоскости и соответствующие им новые количественные критерии. В предлагаемой классификации основными параметрами для разделения нарушений осанки на типы являются HIL ${ }_{\sigma \pm}$, НІК ${ }_{\sigma \pm}-\sigma$-нормированная с коррекцией асимметрии распределений интегральная высота лордоза и кифоза, IHI - индекс асимметрии 
интегральной высоты кифоза (НІК) и лордоза (HIL). Для дифференцирования нормы и субнормы используют также интегральные индексы состояния осанки в сагиттальной плоскости PTI-S (общий индекс), PTI-OS (индекс по ориентации) и PTI-DS (индекс по деформации) [2]. Для дифференцирования сутулой (НО-СуС) и круглой (НО-СуС) спины используют индекс IDLK, определяющий в процентах отклонение границы кифоза и лор- доза от анатомически правильного положения (уровня позвонка $\mathrm{Th}_{12}$ ). Этот индекс отрицателен, если удлинен лордоз, и положителен при удлинении кифоза. Топографические критерии задают с помощью логических выражений, объединяющих отдельные условия по «И» (символ«\&») и «ИЛИ» (символ «»). Объединение двух условий символом «\&» означает, что необходимо одновременное выполнение обоих условий, а для сим- вола «» достаточно выполнение одного из двух условий. Для сложных критериев используют скобки, задающие порядок выполнения логических операции «\&» и «»».

Система топографических критериев, представленная в табл., отработана на более чем 33000 пациентах (КБСОМ), для всех пациентов обеспечила постановку топографического диагноза в сагиттальной плоскости.

\begin{tabular}{|c|c|c|c|c|}
\hline $\begin{array}{l}\text { Табли } \\
\text { Топог } \\
\text { № }\end{array}$ & Обозначение & Наименование & Краткое описание & Топографические критерии \\
\hline 1 & $3 \mathrm{H}$ & 3 доров - норма & Осанка, близкая к гармоничной & $\begin{array}{l}\text { PTI-S }<2 / 3 \& \text { PTI-DS }<1 \& \text { PTI-OS }<1 \\
\& 0,2<\mathrm{IHI}<1,0 \&\left|\left(\mathrm{HIL}_{\sigma \pm}+\mathrm{HIK}_{\sigma \pm}\right) / 2\right|<2 / 3\end{array}$ \\
\hline 2 & 3С-НБ & $\begin{array}{l}\text { Субнорма } \\
\text { с нарушением } \\
\text { баланса }\end{array}$ & $\begin{array}{l}\text { Лордоз и кифоз в норме, нарушен баланс туловища } \\
\text { в сагиттальной плоскости (умеренный или выра- } \\
\text { женный наклон кпереди или кзади) }\end{array}$ & $\begin{array}{l}(\text { PTI-S } \geq 2 / 3 \| \text { PTI-OS } \geq 1) \& \text { PTI-OS }>\text { PTI-DS } \\
\& 0,2<\mathrm{IHI}<1,0 \&\left|\left(\mathrm{HIL}_{\sigma \pm}+\mathrm{HIK}_{\sigma \pm}\right) / 2\right|<2 / 3\end{array}$ \\
\hline 3 & 3С-НГ & $\begin{array}{l}\text { Субнорма } \\
\text { с нарушением } \\
\text { геометрии }\end{array}$ & $\begin{array}{l}\text { Лордоз и кифоз в норме, нарушена геометрия фи- } \\
\text { зиологических изгибов (анатомически правильное } \\
\text { положение их апексов или границы между лордозом } \\
\text { и кифозом) }\end{array}$ & $\begin{array}{l}(\mathrm{PTI}-\mathrm{S} \geq 2 / 3 \| \mathrm{PTI}-\mathrm{DS} \geq 1) \& \mathrm{PTI}-\mathrm{DS} \geq \mathrm{PTI}-\mathrm{OS} \\
\& 0,2<\mathrm{IHI}<1,0 \&\left|\left(\mathrm{HIL}_{\sigma \pm}+\mathrm{HIK}_{\sigma \pm}\right) / 2\right|<2 / 3\end{array}$ \\
\hline 4 & ЗС-Уп & $\begin{array}{l}\text { Субнорма } \\
\text { с уплощением }\end{array}$ & $\begin{array}{l}\text { Дордоз и кифоз сбалансированы и уплощены в } \\
\text { пределах зеленой зоны или один изгиб в зеленой, } \\
\text { а другой - на границе желтой зоны }\end{array}$ & $\begin{array}{l}0<\mathrm{IHI}<1,2 \&\left(-1<\mathrm{HIL}_{\sigma \pm} \leq-2 / 3\right. \\
\&-1<\mathrm{HIK}_{\sigma \pm} \leq-2 / 3 \|-1,1<\left(\mathrm{HIL}_{\sigma \pm}+\mathrm{HIK}_{\sigma \pm}\right) / 2 \leq-0,6 \\
\left.\& \mathrm{HIK}_{\sigma \pm} \leq-0,15 \& \mathrm{HIL}_{\sigma \pm} \leq-0,15\right)\end{array}$ \\
\hline 5 & $3 \mathrm{C}-\mathrm{Уc}$ & $\begin{array}{l}\text { Субнорма } \\
\text { с усилением }\end{array}$ & $\begin{array}{l}\text { Лордоз и кифоз сбалансированы и усилены в преде- } \\
\text { лах зеленой зоны или один изгиб усилен в зеленой, } \\
\text { а другой - усилен на границе желтой зоны }\end{array}$ & $\begin{array}{l}0<\mathrm{IHI}<1,2 \&\left(2 / 3 \leq \mathrm{HIL}_{\sigma \pm}<1\right. \\
\& 2 / 3 \leq \mathrm{HIK}_{\sigma \pm}<1 \| 0,6 \leq\left(\mathrm{HIL}_{\sigma \pm}+\mathrm{HIK}_{\sigma \pm}\right) / 2<1,1 \\
\left.\& \mathrm{HIK}_{\sigma \pm} \geq 0,15 \& \mathrm{HIL}_{\sigma \pm} \geq 0,15\right)\end{array}$ \\
\hline 6 & ЗС-УпК & $\begin{array}{l}\text { Субнорма } \\
\text { с уплощением } \\
\text { кифоза }\end{array}$ & $\begin{array}{l}\text { Лордоз находится в зеленой зоне, а кифоз уплощен } \\
\text { в пределах зеленой или на границе с желтои́ зоной, } \\
\text { при этом уплощение кифоза более выражено, чем } \\
\text { усиление лордоза }\end{array}$ & $\begin{array}{l}\mathrm{IHI} \leq 0,2 \&\left|\mathrm{HIK}_{\sigma \pm}\right|<1,1 \&\left|\mathrm{HIL}_{\sigma \pm}\right|<1 \\
\&\left|\mathrm{HIK}_{\sigma \pm}\right| \geq\left|\mathrm{HIL}_{\sigma \pm}\right|\end{array}$ \\
\hline 7 & $3 \mathrm{C}-\mathrm{Y}_{\mathrm{c}} \boldsymbol{1}$ & $\begin{array}{l}\text { Субнорма с уси- } \\
\text { лением лордоза }\end{array}$ & $\begin{array}{l}\text { Кифоз находится в зеленой зоне, а лордоз усилен } \\
\text { в пределах зеленой зоны или на границе с желтой } \\
\text { зоной, при этом усиление лордоза более выражено, } \\
\text { чем уплощение кифоза }\end{array}$ & $\begin{array}{l}\mathrm{IHI} \leq 0,2 \&\left|\mathrm{HIK}_{\sigma \pm}\right|<1 \\
\&\left|\mathrm{HIL}_{\sigma \pm}\right|<1,1 \&\left|\mathrm{HIL}_{\sigma \pm}\right| \geq\left|\mathrm{HIK}_{\sigma \pm}\right|\end{array}$ \\
\hline 8 & ЗС-УпЛ & $\begin{array}{l}\text { Субнорма } \\
\text { с уплощением } \\
\text { лордоза }\end{array}$ & $\begin{array}{l}\text { Кифоз находится в пределах зеленой зоны, а лордоз } \\
\text { уплощен в пределах зеленой зоны или на границе } \\
\text { с желтой зоной, при этом уплощение лордоза более } \\
\text { выражено, чем усиление кифоза }\end{array}$ & $\begin{array}{l}\mathrm{IHI} \geq 1,0 \&\left|\mathrm{HIK}_{\sigma \pm}\right|<1 \\
\&\left|\mathrm{HIL}_{\sigma \pm}\right|<1,1 \&\left|\mathrm{HIL}_{\sigma \pm}\right| \geq\left|\mathrm{HIK}_{\sigma \pm}\right|\end{array}$ \\
\hline 9 & 3С-УcK & $\begin{array}{l}\text { Субнорма с уси- } \\
\text { лением кифоза }\end{array}$ & $\begin{array}{l}\text { Лордоз находится в пределах зеленой зоны, а кифоз } \\
\text { усилен в пределах зеленой или на границе с желтой } \\
\text { зоной, при этом усиление кифоза более выражено, } \\
\text { чем уплощение лордоза }\end{array}$ & $\begin{array}{l}\mathrm{IHI} \geq 1,0 \&\left|\mathrm{HIK}_{\sigma \pm}\right|<1,1 \\
\&\left|\mathrm{HIL}_{\sigma \pm}\right|<1 \&\left|\mathrm{HIK}_{\sigma \pm}\right| \geq\left|\mathrm{HIL}_{\sigma \pm}\right|\end{array}$ \\
\hline 10 & НО-ПС & Плоская спина & $\begin{array}{l}\text { Лордоз и кифоз уплощены в пределах желтой зоны } \\
\text { или один физиологический изгиб уплощен в пре- } \\
\text { делах желтой, а другой - в пределах зеленой зоны; } \\
\text { или один физиологический изгиб уплощен в преде- } \\
\text { лах красной, а другой - в пределах желтой зоны }\end{array}$ & $\begin{array}{l}-2<\mathrm{HIL}_{\sigma \pm} \leq-1 \&-2<\mathrm{HIK}_{\sigma \pm} \leq-1 \|-1 \leq \mathrm{IHI} \leq 1,3 \\
\&\left(\mathrm{HIL}_{\sigma \pm}+\mathrm{HIK}_{\sigma \pm}\right) / 2 \leq-1 \&\left(\mathrm{HIL}_{\sigma \pm}+\mathrm{HIK}_{\sigma \pm}\right) / 2>-1,5 \\
\&\left(\mathrm{HIL}_{\sigma \pm} \leq-1 \& \mathrm{HIK} \mathrm{K}_{\sigma \pm} \leq-2 / 3 \| \mathrm{HIK}_{\sigma \pm} \leq-1\right. \\
\left.\& \mathrm{HIL}_{\sigma \pm} \leq-2 / 3\right) \|-0,8<\mathrm{IHI}<2,5 \&\left(\mathrm{HIL}_{\sigma \pm}\right. \\
\left.+\mathrm{HIK}_{\sigma \pm}\right) / 2 \leq-1,5 \&\left(\mathrm{HIL}_{\sigma \pm}+\mathrm{HIK}_{\sigma \pm}\right) / 2>-1,75\end{array}$ \\
\hline
\end{tabular}




\begin{tabular}{|c|c|c|c|c|}
\hline № & Обозначение & Наименование & Краткое описание & Топографические критерии \\
\hline 11 & НО-ПВС & $\begin{array}{l}\text { Плосковогнутая } \\
\text { спина }\end{array}$ & $\begin{array}{l}\text { Лордоз усилен в пределах желтой зоны при кифозе, } \\
\text { уплощенном в пределах зеленой и желтой зон, или } \\
\text { при лордозе, находящемся в пределах зеленой зоны, } \\
\text { кифоз уплощен в пределах желтой или красной зоны }\end{array}$ & $\begin{array}{l}\mathrm{IHI} \leq 0 \&\left(\mathrm{HIL}_{\sigma \pm}<2 \& \mathrm{HIL}_{\sigma \pm} \geq 1\right. \\
\left.\& \mathrm{HIK}_{\sigma \pm} \leq-2 / 3 \|-1<\mathrm{HIL}_{\sigma \pm}<1 \& \mathrm{HIK}_{\sigma \pm} \leq-1\right)\end{array}$ \\
\hline 12 & $\mathrm{HO}-\mathrm{BC}$ & Вогнутая спина & $\begin{array}{l}\text { Лордоз усилен в пределах желтой зоны при нормаль- } \\
\text { ном или усиленном в пределах зеленой зоны кифозе }\end{array}$ & $\mathrm{IHI} \leq 0,2 \& 1 \leq \mathrm{HIL}_{\sigma \pm}<2 \& 0 \leq \mathrm{HIK}_{\sigma \pm}<1,05$ \\
\hline 13 & $\mathrm{HO}-\mathrm{KBC}$ & $\begin{array}{l}\text { Кругловогнутая } \\
\text { спина }\end{array}$ & $\begin{array}{l}\text { Лордоз и кифоз усилены в пределах желтой зоны } \\
\text { или один из изгибов усилен в пределах желтой зоны } \\
\text { а другой - в пределах зеленой или красной зоны }\end{array}$ & $\begin{array}{l}0,2<\mathrm{IHI}<1,0 \&\left(1 \leq \mathrm{HIL}_{\sigma \pm}<2 \& 1 \leq \mathrm{HIK}_{\sigma \pm}<2\right. \\
\left.\| 1 \leq\left(\mathrm{HIL}_{\sigma \pm}+\mathrm{HIK}_{\sigma \pm}\right) / 2<2\right) \\
\| 0<\mathrm{IHI}<1,2 \& 1,25 \leq\left(\mathrm{HIL}_{\sigma \pm}+\mathrm{HIK}_{\sigma \pm}\right) / 2<2\end{array}$ \\
\hline 14 & НО-КПС & $\begin{array}{l}\text { Круголоплоская } \\
\text { спина }\end{array}$ & $\begin{array}{l}\text { Лордоз уплощен в пределах желтой или красной } \\
\text { зоны при кифозе, находящемся в пределах зеленой } \\
\text { зоны }\end{array}$ & $1,0 \leq \mathrm{IHI} \& \mathrm{HIL}_{\sigma \pm} \leq-1 \&-1<\mathrm{HIK}_{\sigma \pm}<1$ \\
\hline 15 & HO-CyC & Сутулая спина & $\begin{array}{l}\text { Кифоз усилен в пределах желтои́ зоны и удлинен } \\
\text { по протяженности при уплощенном в пределах } \\
\text { зеленой, желтой или красной зон лордозе }\end{array}$ & $1,0<\mathrm{IHI} \&\left(\mathrm{HIL}_{\sigma \pm}+1<\mathrm{IDLK} / 10\right) \&\left(1 \leq \mathrm{HIK}_{\sigma \pm}<2\right)$ \\
\hline 16 & $\mathrm{HO}-\mathrm{KpC}$ & Круглая спина & $\begin{array}{l}\text { Кифоз усилен в пределах желтой зоны, лордоз нахо- } \\
\text { дится в пределах зеленой или на границе с желтой } \\
\text { зоной, соотношение по протяженности между } \\
\text { лордозом и кифозом не нарушено }\end{array}$ & $1,0<\operatorname{IHI} \&\left(\mathrm{HIL}_{\sigma \pm}+1 \geq \mathrm{IDLK} / 10\right) \&\left(1 \leq \mathrm{HIK}_{\sigma \pm}<2\right)$ \\
\hline 17 & АП-СПС & $\begin{array}{l}\text { Синдром } \\
\text { прямой спины }\end{array}$ & $\begin{array}{l}\text { Лордоз и кифоз уплощены в пределах красной зоны } \\
\text { или один из изгибов уплощен в пределах красной, } \\
\text { а другой - в пределах желтой зоны }\end{array}$ & 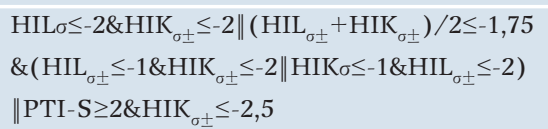 \\
\hline 18 & АП-ГКЛ & $\begin{array}{l}\text { Гиперкифо- } \\
\text { лордоз }\end{array}$ & $\begin{array}{l}\text { Лордоз и кифоз сбалансированы и усилены в преде- } \\
\text { лах красной зоны или один из изгибов усилен в пре- } \\
\text { делах красной, а другой - в пределах желтой зоны }\end{array}$ & $\begin{array}{l}\mathrm{HIL}_{\sigma \pm} \geq 2 \& \mathrm{HIK}_{\sigma \pm} \geq 2 \| 0,2<\mathrm{IHI}<1 \\
\&\left(\mathrm{HIL}_{\sigma \pm}+\mathrm{HIK}_{\sigma \pm}\right) / 2 \geq 2 \& \mathrm{HIL}_{\sigma \pm} \geq 5 / 3 \\
\& \mathrm{HIK}_{\sigma \pm} \geq 5 / 3\end{array}$ \\
\hline 19 & АП-Гл & Гиперлордоз & $\begin{array}{l}\text { Лордоз усилен в пределах красной зоны, кифоз уп- } \\
\text { лощен, соответствует норме или усилен в пределах } \\
2 / 3 \text { желтой зоны }\end{array}$ & $0,2 \geq \mathrm{IHI} \& \mathrm{HIL}_{\sigma \pm} \geq 2,0 \& \mathrm{HIK}_{\sigma \pm}<5 / 3$ \\
\hline 20 & АП-ГК1 & Гиперкифоз I ст. & $\begin{array}{l}\text { Кифоз усилен в пределах красной зоны ( от } 2 \text { до 3-х б), } \\
\text { лордоз уплощен, соответствует норме или усилен }\end{array}$ & $1,0 \leq \mathrm{IHI} \& 2 \leq \mathrm{HIK}_{\sigma \pm}<3 \& \mathrm{HIL}_{\sigma \pm}<2$ \\
\hline 21 & АП-ГК2 & Гиперкифоз II ст. & $\begin{array}{l}\text { Кифоз усилен в пределах красной зоны (более } 3 \text {-х б), } \\
\text { лордоз уплощен, соответствует норме или усилен }\end{array}$ & $1,0 \leq \mathrm{IHI} \& 3 \leq \mathrm{HIK}_{\sigma \pm} \& \mathrm{HIL}_{\sigma \pm}<2$ \\
\hline
\end{tabular}

На рис. 5 приведены сагиттальные профили (линии остистых отростков позвоночника при виде сбоку) для 21 типа нарушений осанки предлагаемой классификации: верхний ряд профилей соответствует зеленой зоне, второй ряд - желтой, третий - красной.

На рис. 6 представлена структура нарушений осанки по типам, полученная по КБСОМ, отдельно для мужского и женского пола. Для зеленой зоны: у мальчиков чаще встречается два типа нарушений с превалированием кифоза (ЗС-УпЛ - 11,93\% и ЗС-УсК - 11,46\%), у девочек наблюдается более равномерная структура по типам нарушений. Для желтой зоны выявлены более существенные различия структуры нарушений по полу: у мальчиков превалируют нарушения осанки с преобладанием кифоза (НО-КПС - 13,03\%, НО-КрС - 8,17\%), у девочек - плосковогнутая спина, нарушение осанки с преобладанием лордоза (НО-ПВС - 19,03\%). Подобная картина наблюдается и для красной зоны, где у мальчиков превалирует гиперкифоз (ДП-ГК1-2,46\%, ДП-ГК2-0,30\%), у девОчек - гиперлордоз (ДП-ГЛ - 2,79\%). Следует отдельно отметить тот факт, что вся красная зона в процентном отношении суммарно для мальчиков и девочек составила 4,55\%, что хорошо согласуется с нормальным законом распределения, при котором для значений более $\pm 2 \sigma$ процент случаев составляет 4,56\%. Это может служить доказательством обоснованности коррекции асимметрии распределений топографических параметров, которая была использована в рамках настоящего исследования. Для старых формул (1) и (2) без этой коррекции количество пациентов красной зоны по КБСОМ составляло около $10 \%$. 


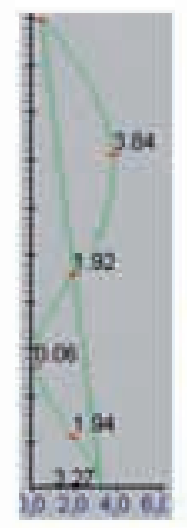

1

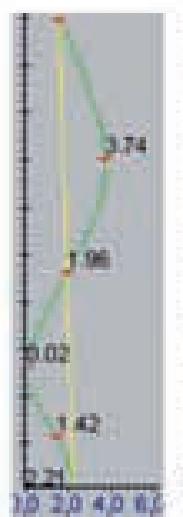

2

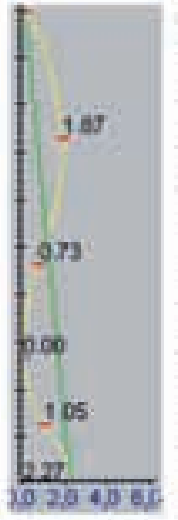

10

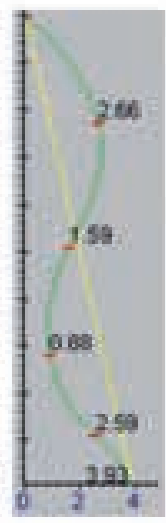

3

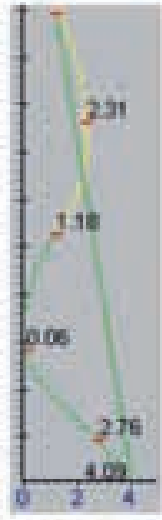

11

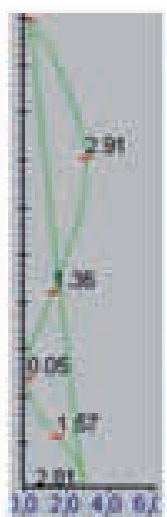

4

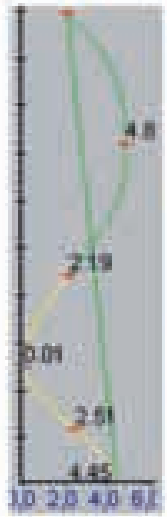

12

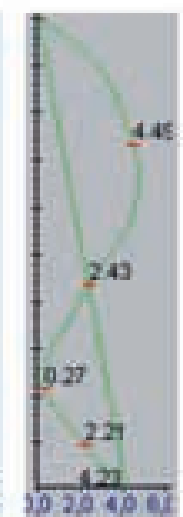

5

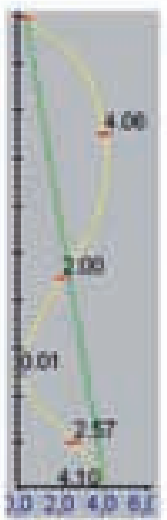

13

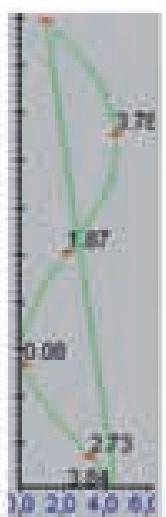

6

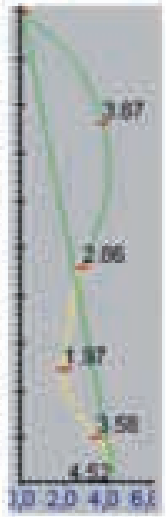

14

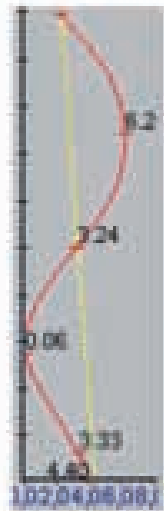

18

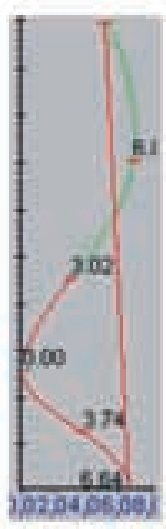

19

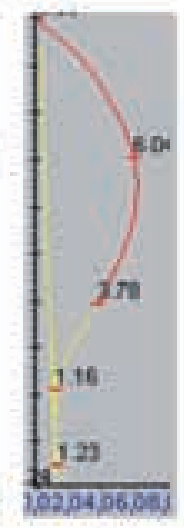

20
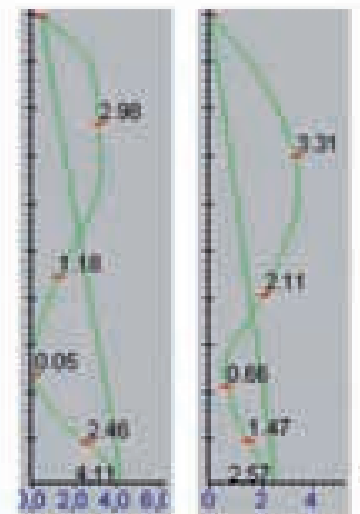

7
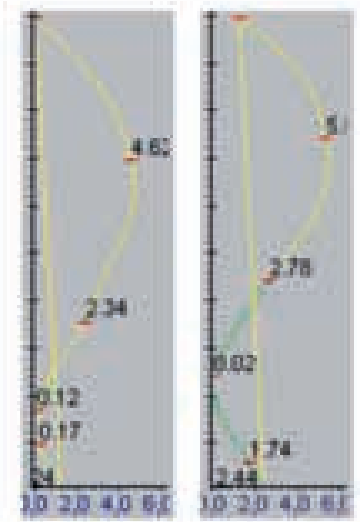

15

16

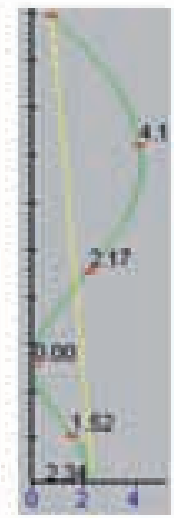

9

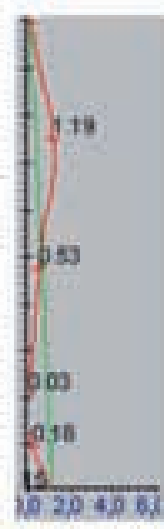

17

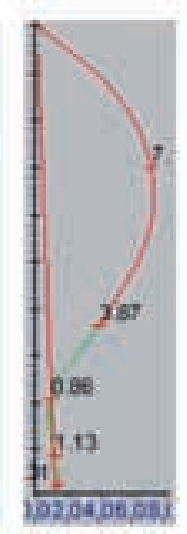

21

Рис. 5

Примеры сагиттальных профилей для типов нарушений осанки в сагиттальной плоскости с нумерацией (1-21) в соответствии с табл. 


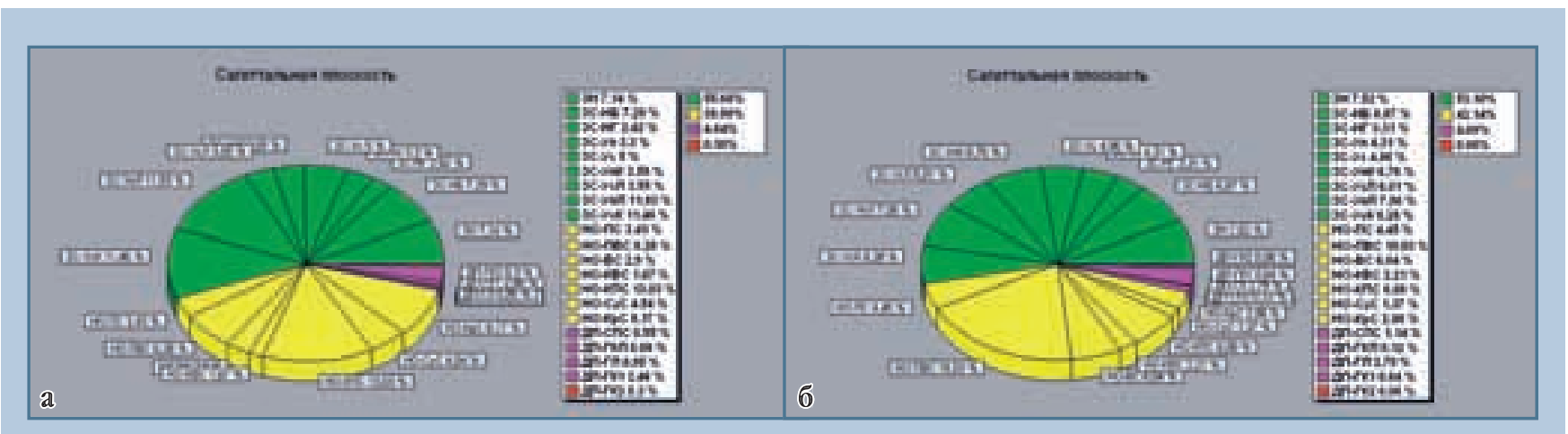

Рис. 6

Структура нарушений осанки в сагиттальной плоскости пациентов 4,5-17,5 лет мужского (а) и женского (б) пола

\section{Заключение}

Предложена новая классификация нарушений осанки в сагиттальной плоскости по данным метода КОМОТ, созданная на основе рассчитанных по базе статистических параметров и переработанных количественных топографических критериев. Общее число обследованных в шести регионах более 33000 пациентов 4,5-17,5 лет.

Классификация позволяет дифференцировать нарушения осанки по тяжести на слабовыраженные, умеренно выраженные и выраженные отклонения. Она содержит 21 тип осанки: гармоничная осанка (3Н), 8 типов осанки для слабовыраженных нарушений (3С), 7 - для умеренных (НО),
5 - для выраженных (ДП). Классификация учитывает все возможные варианты соотношения по выраженности физиологических изгибов: сбалансированное уплощение (ЗС-УП $\rightarrow$ НО-ПС $\rightarrow$ ДП-СПС); сбалансированное усиление (ЗС-УС $\rightarrow$ HО-КВС $\rightarrow$ ДП-ГКЛ); усиление лордоза при нормальном кифозе (ЗС-УсЛ $\rightarrow$ НО-ВС $\rightarrow$ ДП-ГЛ); усиление лордоза при уплощенном кифозе (ЗС-УПК $\rightarrow$ НО-ПВС $\rightarrow$ ДП-ГЛ); усиление кифоза при нормальном лордозе $($ ЗС-УсК $\rightarrow$ HO-КрС $\rightarrow$ ДП-ГК $1 \rightarrow$ ДП-ГК2); усиление кифоза при уплощенном лордозе (ЗС-УПЛ $\rightarrow$ НО-КПС $\rightarrow \mathrm{HO}-\mathrm{CуC} \rightarrow$ ДП-ГК1 $\rightarrow$ ДП-ГК2). Для сбалансированного состояния лордоза и кифоза с нормой по выраженности дополнительно выделяют- ся субнорма с нарушением баланса (ЗС-НБ - отклонение кпереди-кзади) и субнорма с нарушением геометрии (ЗС-НГ - отклонение от правильного анатомического положения апекса лордоза и кифоза или границы между ними).

Новая классификация позволяет более точно дифференцировать состояние осанки в сагиттальной плоскости по результатам обследований на системе ТОДП, что важно как для клинической практики, так и для научных исследований. В дальнейшем с ее помощью планируется провести исследование половозрастных особенностей осанки с оценкой возможных региональных различий.

\section{Литература}

1. Евразийский пат. № 000111. Способ компьютерной оптической топографии тела человека и устройство для его осуществления / Сарнадский В.Н., Садовой М.А., Фомичев Н.Г;; заявл. 26.08.96; опубл. 27.08.1998, Бюл. № 4.

2. Сарнадский В.Н., Фомичев Н.Г., Садовой М.А. Мониторинг деформации позвоночника методом компьютерной оптической топографии. Новосибирск, 2001.

3. Сарнадский В.Н. Формализованный топографический диагноз по результатам скрининг-обследования пациентов в 4 позах // Диагностика, профилактика и коррекция нарушений опорно- двигательного аппарата у детей и подростков: Тез. докл. II Всерос. науч.-практ. конф. с междунар. участием. М., 2008. С. 153-156.

\section{References}

1. Sarnadskiy VN, Sadovoy MA, Fomichev NG. [Method of Computer Optical Topography of the Human Body and Device for its Implementation]. EA Patent 000111, filed 26.08.1996, publ. 27.08.1998. In Russian.

2. Sarnadskiy VN, Fomichev HG, Sadovoy MA. [Monitoring of Spinal Deformity Using Computer Optical Topography]. Novosibirsk, 2001. In Russian.
3. Sarnadskiy VN. Formalized topographic diagnosis based on screening examination of patients in 4 poses]. Diagnosis, prevention, and correction of the musculosceletal system disorders in children and adolescents. Proceedings of the Conference, Moscow, 2008:153-156. In Russian.

Адрес для переписки:

Сарнадский Владимир Николаевич 630091, Новосибирск, ул. Крылова, 31, оф. 54 , OOO «METOC», metos.org@gmail.com

Статья поступила в редакцию 10.05.2011 